\title{
El papel del profesor-asesor en el prácticum de la Licenciatura en Educación Física, Recreación y Deporte*
}

\author{
Carlos Federico Ayala-Zuluaga ${ }^{1}$ \\ Cristian Camilo Orozco-Sánchez ${ }^{2}$
}

Recibido: 09-02-2020

Aceptado: 06-05-2020

\section{Resumen}

Este artículo está basado en resultados de los proyectos investigativos "Orientações pedagógicas no estágio supervisionado de educação física na Colômbia, Brasil, Argentina e Chile" y "Sentidos de los modos corporales y saberes docentes del prácticum en Educación Física, Recreación y Deporte de la Universidad de Caldas", motivados por el interés de comprender el papel y las características del profesorasesor en la formación del practicante-profesor, en el contexto del prácticum de la Licenciatura en Educación Física, Recreación y Deporte. Se trata de un diseño investigativo apoyado en el enfoque cualitativo, de corte histórico-descriptivo-hermenéutico, con estudio de caso múltiple, técnicas de entrevista en profundidad y análisis documental. En este contexto, los resultados presentados se centran en el rol de profesor-asesor desde la reflexión, acción,

\footnotetext{
*Este texto expone los resultados de dos proyectos investigativos en Colombia y Brasil, y corresponde a la categoría de artículo de investigación científica y tecnológica.

1. Licenciado en Educación Física y Recreación; magíster en Educación; doctor en Ciencias de la Motricidad. Docente asociado, Universidad de Caldas, Manizales, Colombia; Grupo Cumanday Actividad Física y Deporte; Licenciatura en Educación Física, Recreación y Deporte.

Correo electrónico: federico.ayala@ucaldas.edu.co

ORCID: http://orcid.org/000-0001-6144-4535

Google Scholar: https://scholar.google.es/citations?user=4bhagxkAAAAJ\&hl=es
}

2. Licenciado en Educación Física, Recreación y Deporte; magíster en Educación. Docente, Universidad de Caldas, Manizales, Colombia; Grupo Cumanday Actividad Física y Deporte; Licenciatura en Educación Física, Recreación y Deporte.

Correo electrónico: cristian.orozco@ucaldas.edu.co

ORCID: https://orcid.org/0000-0002-9832-0808

Google Scholar: https://scholar.google.com/citations?hl=es\&authuser=1 \&user=4bJ WbUQAAAAJ 
orientación, asesoría y relación con actores del prácticum, para aportar en el conocimiento de las prácticas docentes, el rol de profesor-asesor y la visión de los practicantes-profesores sobre este, y cómo se articulan saberes docentes facilitando acciones didácticas y fortaleciendo la identidad docente.

Palabras clave: didáctica, licenciatura, prácticum, educación superior, escuela.

\title{
The role of the teacher-advisor in the practicum of the Degree in Physical Education, Recreation and Sport
}

\begin{abstract}
This article is based on the results of the research projects "Pedagogical Orientations are not supervised in physical education in Colombia, Brazil, Argentina and Chile" and "Senses of the body modes and teaching knowledge of the Practicum in Physical Education, Recreation and Sport of the University de Caldas ", motivated by the interest in understanding the role and characteristics of the teacher-advisor in the training of the practitioner-teacher, in the context of the Practicum of the Degree in Physical Education, Recreation and Sport. It is an investigative design supported by the qualitative approach, of a historical-descriptivehermeneutic nature, with a multiple case study, in-depth interview techniques and documentary analysis. In this context, the results presented focus on the role of teacher-advisor from reflection, action, orientation, advice and relationship with practitioner actors, to contribute to the knowledge of teaching practices, the role of teacher-advisor and the view of the practitioners-teachers on this, and how teaching knowledge is articulated facilitating didactic actions and strengthening the teaching identity.
\end{abstract}


Keywords: didactics, bachelor's degree, practicum, higher education, school.

\section{Introducción}

Los procesos de formación docente en los programas de Licenciatura en Educación Física, Recreación y Ciencias del Deporte, desde finales del siglo XX hasta la actualidad, han intensificado la búsqueda de coadyuvar en la formación inicial de los futuros licenciados en los campos de la educación, la didáctica, la pedagogía específicamente para el deporte y la actividad física, según los cambios de orden mundial en lo económico, educativo y formativo respecto a las dinámicas y políticas públicas, el uso de las tecnologías, el comportamiento económico de la región y los costumbres culturales específicas para cada época.

Los países abordados en las investigaciones referenciadas, presentan diversas posturas que adopta el profesor-asesor ${ }^{3}$ en los programas curriculares establecidos en las instituciones de educación superior, es por ello, que se afronta un gran dilema respecto a la calidad educativa y asi el actor educativo cumple o no con los objetivos de formarse y ser formador de los practicantes-profesores $^{4}$, pues se cree que entre mayor y mejor sea el proceso formativo pedagógico y profesional será posible una mejor calidad educativa (Bolívar, 2019).

Por eso, a finales del siglo XX y en lo que va del XXI, los procesos formativos en el campo de la educación física, recreación y deporte en el Brasil se han convertido en políticas públicas para la población, las cuales brindan posibilidades deportivas, recreativas,

3. El profesor-asesor es aquel que funge como profesor universitario y es el responsable de orientar o asesorar al practicante-profesor en el proceso del prácticum.

4. El practicante-profesor en Colombia y Brasil es un concepto que se refiere al estudiante universitario que se enfrenta al proceso del prácticum y que funge como estudiante y como profesor en propiedad, ya que algunas de sus funciones se asemejan a las del profesor-titular, tales como la planeación, estructuración, evaluación, investigación y reflexión sobre las experiencias teórico-prácticas en la clase de educación física. 
didácticas, educativas y de formación de talento humano para los nuevos desafíos en este campo(Gómez \& Ostos, 2018).

El prácticums ${ }^{5}$ en el Brasil, inició en el año 1977 con la Ley n. ${ }^{\circ}$ 6494, que dispone las prácticas de estudiantes en establecimientos de enseñanza superior y profesional por quienes cumplieran con las habilidades para ejercer dicha práctica, tal espacio didáctico era denominado "Estágio Curricular Supervisionado." (Presidência da República, 1977). En 1987, hay una reestructuración curricular de los cursos sobre las generalidades filosóficas (filosofía de la educación, etc.), sociales (historia de la educación física, etc.) y del ser humano (biología, anatomía, biomecánica, etc.), y en el conocimiento técnico se abordaron aspectos sobre la didáctica, la práctica de enseñanza, el currículo, etc; además, la apropiación del conocimiento en actividades de entrenamiento deportivo, comunicación en educación física, deportes comunitarios, estudio del profesional de educación física como agente del saber, etc. (Souza Neto, 2014; Simões y Silva, 2013).

Con lo anterior, se encontró la necesidad de incluir en los planes curriculares un profesor-asesor o formador acompañante que se dispusiera a "coordinar, planear, programar, supervisionar, dinamizar, dirigir, organizar, evaluar y ejecutar trabajos, programar proyectos, prestar servicios de auditoría, consultoría y asesoría [...], elaborar informes técnicos, científicos y pedagógicos, todos en áreas de actividades físicas y del deporte" (Presidência da República, 1998, p. 1), articulando la teoría y la práctica, como inmersión del saber profesional y el saber reflexionar sobre dicha práctica (Schön, 2002); esto permitió la relación de la universidad con la escuela, a través de convenios académicos y de formación (Conselho Nacional de Educação, 2002).

En la época contemporánea, respecto a la formación de docentes en Brasil en el campo de la educación física, recreación y deporte, su modelo curricular es enfocado a aspectos académicos, mecanismos de práctica y de experimentación, a partir de las expe-

5. Según Zabalza (2006), "el prácticum constituye una parte importante en la carrera profesional, ya que se integra y se enriquece el conocimiento del estudiante en su proceso de formación académico (teórico-práctico) con la experiencia en los diversor escenarios de aprendizaje en los centros de trabajo" (p.314). 
riencias didácticas en el campo profesional denominado práctica supervisionada (Fuzii, 2010). Finalmente, en la Ley 11.788 del 2008, se establecen las directrices educativas en el prácticum respecto a lo teórico y lo práctico, como medio de mejoramiento de la calidad educativa en los procesos de enseñanza y aprendizaje en el campo de conocimiento (Presidência da República, 2008).

En Colombia, la formación de los maestros ha pasado por diferentes momentos históricos desde la época precolombina, con los indígenas, la conquista y colonización española en América, la formación militar en aspectos de defensa del territorio para la protección de la población; en los siglos XVII y XVIII, en aspectos ideológicos, militares, intelectuales y didácticos; en el siglo XIX, con ideales froebelianos (José Manuel Marroquín) centrados en la sociedad y la naturaleza; en las guerras civiles (1888-1892); en el siglo XX (1914 hasta 1994), con la Ley 115, se priorizó la educación infantil en la etapa preescolar en todas las instituciones educativas; finalmente, con la Resolución 18583 del 2017 se fortalecieron las competencias en la formación pedagógica, en las TIC y en el dominio de un segundo idioma (Ávila, 2019, Ministerio de Educación Nacional [MEN], 2017; Ocampo, 2009; Ley 115 de 1994).

La formación de educadores en el campo especifico de la educación física, recreación y deporte en Colombia inicialmente fue asumida por personas apasionadas por las actividades deportivas, que basaron sus conocimientos en la adquisición de habilidades, competencias y experticias (Acosta y Vasco, 2013); este panorama cambió con la creación del INEF (Instituto Nacional de Educación Física) como punto de partida en la formación de formadores para esta área en Colombia, apoyados en el enfoque tradicional o tecnicista ${ }^{6}$ con perspectivas en las que el maestro impartía el conocimiento y los estudiantes escuchaban (Portela, Taborda y Loaiza, 2017), visión que ha cambiado con las nuevas tendencias críticocognitivistas de la educación.

Específicamente, los procesos del prácticum (o prácticas profesionales docentes [PPD]) desde sus inicios se enfocaron en las 
tendencias "fisicalistas" (educar en y a través del movimiento), lo cual ha conducido a realizar actualizaciones curriculares constantes frente a lo educativo, pedagógico, deportivo y didáctico (Betancur, López y Arcila, 2018); luego se empezó a concebir la totalidad del individuo (en sus aspectos social, económico, espiritual, académico, de rendimiento físico y deportivo, entre otros) como "manifestación de una unidad que crea permanentemente la existencia, que tiene saberes y los recrea" (Taborda, 2017, p. 196), y en el cual se representan simbolismos laborales, que el practicanteprofesor asume con conciencia y profesionalidad, desde las experiencias, las acciones y los aprendizajes (Martínez, 2019).

Ahora bien, el prácticum o práctica profesional docente (PPD), en sus componentes pedagógicos y didácticos (metodología, contenidos curriculares, evaluación, entre otros), busca afianzar los conocimientos y múltiples saberes adquiridos en la formación (Zabalza, 2013; MEN, 2017; Ley 115 de 1994), en un proceso en que el practicante-profesor en educación física construye sus saberes docentes a partir de la reflexión en la acción; el reconocimiento del contexto, las costumbres culturales, la historia racional de cada época, de los dispositivos de poder y de cómo estos funcionan en lo educativo, así como de los modos específicos de ser de la historia en ciertos momentos (Cárdenas y Cárdenas, 2019). Esto se logra a partir del profesor-asesor (formador de formadores) cuya función es acompañar en los aspectos didácticos y pedagógicos durante el prácticum para que se desarrollen los diferentes saberes (Tardif, 2010); además, Ayala, Souza y laochite (2017) consideran que el profesor-asesor en el prácticum guía al practicanteprofesor en el proyecto político pedagógico (PPP), cuya

práctica docente tiene como objetivo central desarrollar en los licenciandos la capacidad de involucrarse e intervenir científica, humana y pedagógicamente en las instituciones y en las comunidades educativas, mediante el ejercicio docente [...] Por tanto, se entiende que la formación de los alumnos se desenvuelve en torno a lo que se denomina Modelo Cognitivo- Experiencial - Reflexivo, en el cual se busca indagar y comprender los actos edu- 
cativos, pedagógicos y didácticos en la práctica docente y demostración del ejercicio docente. (p. 205)

Ahora bien, el prácticum en Colombia y Brasil se han estructurado bajo la premisa de la relación entre la práctica y la teoría como praxis, las cuales vinculan, recrean e inducen a repensarlo desde los conocimientos, los saberes y el papel del profesor-asesor en el proceso; de este modo, el problema en ambas investigaciones surge a partir de querer superar los desafíos que se presentan respecto a la insuficiente calidad para la formación integral de los sujetos y del contexto educativo; y al reclamo de los practicantesprofesores ante las asesorías y visitas institucionales, la comunicación entre universidad y escuela, la escasa retroalimentación en las prácticas y la relación jerárquica del profesor-asesor con el practicante-profesor durante el prácticum.

\section{Metodología}

El proceso metodológico se centró en el enfoque cualitativo, con el fin de comprender e interpretar los aspectos intrínsecos, subjetivos y de la historicidad del practicante-profesor durante el prácticum en la Universidad Estadual Paulista en Brasil, práctica que es denominada Estágio Supervisionado, y en la Práctica Profesional Docente en la Universidad de Caldas en Colombia. En concordancia con lo anterior, se aborda la investigación cualitativa, ya que es la que se encarga de indagar en profundidad de manera holística los

valores, creencias, hábitos, actitudes, representaciones y opiniones, y se adecua a profundizar a complejidad, los datos y procesos particulares y específicos de los individuos y grupos. El enfoque cualitativo es empleado, por tanto, para la comprensión de fenómenos caracterizados por un alto grado de complejidad interna. (Paulilo, 1999, p. 135) 
El método empleado fue el histórico-descriptivo-hermenéutico, en un proceso que se realizó en un momento determinado, específico y real, permitiendo representar y simbolizar la información recaudada a través de la triangulación de la información para luego analizarla, interpretarla y comprenderla en torno al fenómeno estudiado. También se utilizó el estudio de caso múltiple, ya que se buscó establecer las particularidades, complejidades, limitaciones, características y procedimientos de las universidades y programas a través de la confrontación de los discursos.

En los resultados obtenidos se basó en el procedimiento de Wood y Smith (2018), el cual recoge las respuestas de los actores entrevistados como fuente de información; seguido a ello, estas fueron reducidas a unidades de análisis, a partir de la codificación de la información en categorías para construir conocimiento mediante el cuerpo teórico (André, 2013).

En esta perspectiva, metodológicamente se consideraron los siguientes puntos en las investigaciones:

- El componente de análisis fueron la Universidad Estadual Paulista en Brasil y la Universidad de Caldas en Colombia, instituciones encargadas de ofrecer programas de formación docente, específicamente los programas de Licenciatura en Educación Física, Recreación y Deporte.

- El componente o unidad de trabajo: es el practicanteprofesor que valora el rol del profesor-asesor (formador de formadores; supervisor en Brasil) del programa, cuya tarea, en palabras de Freire (1994), se concibe así: "la tarea del docente, que también es aprendiz, es placentera y a la vez exigente. Exige seriedad, preparación científica, física, emocional y afectiva" ( $p$. 8); de esta manera, se fortalecen los saberes docentes 
en las prácticas docentes del formador y del que es formado.

\section{Procedimiento}

Inicialmente, se hizo un acercamiento con los programas académicos, seguido a lo cual se solicitó un encuentro en un lugar específico con la disposición y tiempo de los practicantes-profesores que cursaban la PPD y el Estágio Curricular Supervisionado (ECS), quienes fueron invitados a participar voluntariamente para responder al proceso de la entrevista en profundidad (Díaz, Torruco, Martínez y Varela, 2013)', con una duración aproximada de 40 a 60 minutos. Las entrevistas fueron grabadas en un dispositivo móvil para su posterior transcripción.

Según el diseño de la investigación, en primer lugar los investigadores idearon supuestos sobre diferentes problemáticas acontecidas en la PPD y en el ECS, que se desarrollaban en los contextos escolares, que enfrentaba los practicantes-profesores y el profesorasesor en el prácticum en Educación Física, Recreación y Deporte; luego se les indagó y se confrontó a estos con los supuestos planteados para finalmente definir y resolver el(os) problema(s) investigativo(s).

En virtud de lo anterior, se indagó a los practicantes-profesores sobre las problemáticas vivenciadas, con el apoyo de instrumentos como los cuestionarios estructurados (entrevista en profundidad), diarios de campo y grabadora de sonido. Toda la información recolectada fue codificada mediante el programa científico Atlas.ti (versión 7), a través de la codificación abierta, axial y selectiva (Strauss y Corbin, 2002), y se usó la sabana de datos para construir las diferentes categorías en relación al problema y los objetivos planteados.

\footnotetext{
8. Según Díaz et al., esta es una "técnica utilizada desde la antigüedad, no por ello menos actual, de hecho, con las nuevas tecnologías se potencia cada parte del proceso desde el inicio, desarrollo hasta su análisis. La entrevista es uno más de los instrumentos cuyo propósito es recabar datos, pero debido a su flexibilidad permite obtener información más profunda, detallada, que incluso el entrevistado y entrevistador no tenían identificada, ya que se adapta al contexto y a las características del entrevistado" (p. 166).
} 
Finalmente, luego del análisis minucioso de la triangulación de la información se presentó el informe final confrontando la información obtenida con las evidencias empíricas y teóricas en los documentos y las interpretaciones (hermenéutica) de los investigadores, para así construir nuevo conocimiento.

\section{Discusión}

Con base en los análisis de la información obtenida en ambas investigaciones en el contexto del prácticum y el rol del profesor-asesor en los programas de Licenciatura en Educación Física, Recreación y Deporte, se resalta la importancia del practicante-profesor y el formador (asesor o supervisor educativo) durante las sesiones de clase, y en la comprensión e interpretación de los procesos didácticos basados en los componentes teórico/prácticos que se llevan a cabo en el prácticum, además de los aspectos que contribuyen al mejoramiento, desarrollo y acompañamiento de las prácticas en la relación universidad-escuela.

A través de las vivencias en la PPD y el ECS se contribuye a que el maestro en formación edifique el saber, saber hacer y saber ser, lo cual estructura la identidad docente y las orientaciones pedagógicas (MEN, 2010), de acuerdo a las necesidades y situaciones presentadas en el contexto; por ello, el prácticum, como mecanismo de trasformación en el campo educativo de la educación física, se debe asumir con visión analítica, crítica y propositiva, para que le afiancen todos los campos del saber.

Bien se sabe que el prácticum en el sistema educativo depende de las políticas educativas establecidas, sin embargo, Lobo y Quintero (2016) manifiestan que "en América Latina la supervisión educativa se ha convertido en uno de los procesos más cuestionados y condicionados principalmente por trámites administrativos que lo obligan a dejar de lado aspectos pedagógicos cruciales para el desarrollo de la educación" (p. 105). 
En concordancia con lo anterior, los procesos educativos en Brasil y Colombia se sustentan en el desarrollo valorativo de estándares cuantitativos parametrizados, haciendo referencia al fortalecimiento de competencias y al alcance de altos rendimientos educativos; sin embargo, esto no es lo más adecuado para orientar los procesos que fomenten la reflexión y trasformación de la universidad, la escuela y la sociedad, específicamente desde las distintas prácticas pedagógicas y docentes que se puedan llevar a cabo, en este caso, en la clase de educación física como lo entienden Corrales, Ferrari, Gómez y Renzi (2010), pues esta es una

\begin{abstract}
disciplina pedagógica que contribuye a la construcción de la corporeidad y motricidad, con fuerte consideración de los contextos socioculturales en los que ejerce su acción, en constante reflexión sobre su propia identidad disciplinar y sobre las estrategias de intervención necesarias para que dicha contribución sea posible. (p. 29)
\end{abstract}

Según lo anterior, en el área de la educación física el profesor-asesor es el encargado de que se incluyan en los procesos de acompañamiento la autocrítica, comprensión, formación y colaboración en la utilización de los saberes docentes, para que el aula de clases sea un espacio o escenario de actuación, emancipación y libertad de expresión que apueste por la enseñanza y el aprendizaje (Martínez y Raposo, 2010; Correa, 2011; Ayala, Souza y laochite, 2015; Saiz y Susinos, 2016), y por que el sujeto tenga trascendencia e imaginación conectada con la realidad, que perciba los contextos más allá de su cuerpo (Ayala, Souza y laochite, 2015). Pero también pretende que sea un espacio para reflexionar sobre la pertinencia del proceso y de los discursos de los actores, del papel otorgado a la formación y a los formadores (Bolívar, 2019), y del desarrollo investigativo y la implementación de estrategias hacia la adquisición de aprendizajes y enseñanzas constructivas (Martínez, 2019). Según palabras de practicantes-profesores, el profesor-asesor permite o fomenta la confrontación teórica y práctica de los conocimientos, contribuye al desarrollo de la identidad docente y a mirar las cosas desde una postura más profesional: 
Eu acredito que para mim foi muito bom a disciplina de estágio curricular supervisionado, porque conseguimos confrontar mesmo essa parte da teoria com a prática e o ponto de vista que eu tenho é que está muito longe a teoria da prática. (Entrevista 4 a Practicante-profesor en la Universidad Estadual Paulista, 12/08/2014)

El asesor me ayudó a reflexionar sobre las experiencias que uno haya tenido en el trasegar de la vida y no solamente en la vida académica, sino en la vida laboral, como estudiantes en la universidad, cuando estábamos en el colegio, que un profesor nos enseñó esto, lo otro $[\ldots]$, entonces por eso en la práctica tiene que ver mucho la experiencia. (Entrevista 1 a practicante-profesor en la Universidad de Caldas, 12/02/2018.)

El profesor-asesor debe reflexionar sobre sus vivencias (Schön, 2002), a través de sus interacciones teórico/prácticas con los practicantes-profesores que estén realizando el prácticum, ya que la formación favorece la integralidad tanto a nivel personal como profesional, por lo cual en los procesos de asesoramiento es importante tener en cuenta la formación inicial del profesorado (Herrera, Buitrago y Ávila, 2016), el aprendizaje significativo (Ausubel, 2002), desde la reproducción de lo que han experimentado cuando ocupaban el rol de practicante-profesor (Tardif, 2010), y la formación emocional (Torrijos, Martín y Rodríguez, 2018).

En tal efecto, si no existiera el profesor-asesor, no habría procesos de reflexión en el prácticum sobre lo adquirido en la universidad, y sobre el contexto escolar y social. En consecuencia, tanto la universidad como la escuela han de transformarse a la par, ya que los actores educativos se enfrentan a realidades escolares y a un mundo en constante transformación según las políticas de la Nación y de la globalización. Frente a esto cabe resaltar que el profesor-asesor (formador de formadores) debe tomar las mejores decisiones teórico/prácticas (sobre conocimientos epistemológi$\cos$ y experiencias pedagógicas-didácticas) con los practicantesprofesores; en tal sentido, se expresa que: 
La asesora ha sido de gran apoyo, un polo a tierra en muchos aspectos, desde el crecimiento personal del abordaje conceptual, metodológico, pedagógico y didáctico, pienso que sus intervenciones han sido muy buenas, puesto que nos brindan pautas para saber cómo hacer este tipo de abordajes pedagógicos en el colegio, realmente no hubiera sido posible sin la asesora, esto es un trabajo conmensurado, de apoyo mutuo donde doy gracias porque ha estado presente en todo este proceso y de buena manera, ya que no solamente es estar, sino hacerse partícipe, ella ha sido muy partícipe en mi proceso. (Entrevista 7 a practicante-profesor en la Universidad de Caldas, 15/04/2018)

[...] tive um envolvimento muito grande com o professor da disciplina e eu consegui entender muitas coisas que ele falava, gostava da leitura dos textos, sempre li os textos da disciplina e achava muito interessante as coisas que eram suscitadas. Então para mim foi muito bom isso na questão da minha identidade, eu pude conhecer diversas maneiras de se fazer a mesma coisa, podia entender a situação do professor dentro da escola, é como se você desse um zoom naquela situação, e começasse a ver de fora, você está lá dentro, mas você começa a ver de fora, não sei se você consegue entender e isso foi uma coisa que o estágio me propiciou. (Entrevista 3 a practicante-profesor en la Universidad Estadual Paulista, 06/08/2014)

Se resalta que en el transcurso del prácticum la función del profesor-titular o supervisor de la escuela, que consiste en apoyar los aspectos conceptuales, metodológicos, pedagógicos y didácticos; además de ello, el profesor-asesor conduce al practicante-profesor a comprender la práctica con apoyos comunicativos a través de consejos, recomendaciones, concepciones teóricas planteadas en libros, artículos investigativos, entre otros, todos referentes al contexto educativo. 
Para Saiz y Susinos (2016), las funciones principales del profesortitular' son:

Coordinar y animar el debate y la reflexión conjunta, promover la reflexión individual y grupal, proponer medios y recursos para la reflexión, mantener un modo colaborativo de acompañamiento, generar un espacio confiado y seguro para la reflexión y soporte emocional y mantenimiento de la unidad de grupo. (p. 60)

Por tal razón, una adecuada relación entre el profesor-asesor y el profesor-titular tendrá incidencia en el proceso del prácticum y en el practicante-profesor, desde la construcción y formación de saberes docentes (Tardif, 2010), en la formación inicial (Herrera, Buitrago y Ávila, 2016), la educación emocional (Bisquerra, 2016), la autonomía docente (Santos, 2006), y la vigilancia, control y fiscalización (Soler, 2001); así pues, es importante que el asesor o el supervisor sea más expresivo, respetuoso, automotivado y empático, aspectos que optimizan su labor educativa (Calle, Remolina y Velásquez, 2011, p.105).

Lo cierto es que el profesor-asesor, en el caso de las licenciaturas en Educación Física, Recreación y Deporte en Colombia y Brasil, deberá tener adecuados conocimientos en los aspectos conceptuales, pedagógicos, didácticos, deportivos, recreativos, y sobre las nuevas tendencias (productivas, tecnológicas, políticas y sociales), con el fin de dar recomendaciones al practicante-profesor durante el prácticum, específicamente en lo referente a la complejidad de los contextos y los procesos de enseñanza y aprendizaje en las instituciones educativas.

Sin embargo, los practicantes-profesores sienten y expresan que los profesores-asesores durante el prácticum no disponen de un alto interés de acompañar este proceso en el espacio escolar, y que le dan poca importancia al proceso educativo del estudiantado en el desarrollo didáctico, ya que se enfocan más en los proce- 
sos evaluativos y de calificaciones según las directrices o políticas gubernamentales e institucionales. En relación con lo anterior, los estudiantes expresan que:

Pensaba en cancelar la práctica por él (asesor), porque a mí jamás se me acercó a decirme: ¿usted cómo da la clase?, ¿qué hace para que las niñas le hagan silencio? Jamás. Él era: necesito el proyecto, para tal fecha hay que mandarlo, pues lo otros sí le exigían a él, entonces él nos empezaba a apretar a nosotros, pero yo le apuesto que él nunca se leyó el proyecto mío. [...] cierto día llegó a verme una clase, y yo no tenía la clase impresa y me dijo: yo no le veo así la clase, ¿usted no tiene el plan de clase impreso? Entonces yo le dije: profe, pero yo lo tengo acá en el computador, y me dijo: ¡no!, ¡no!; vea que usted me hace venir a perder el tiempo [...]. Entonces yo le decía a él: profe a mí me gustaría, así sea que usted no me vaya a evaluar, quédese para que usted vea la clase [...], y él me dijo: ¡no!, ¡no!; yo me tengo que ir porque yo así no le veo la clase, y se fue. (Entrevista 1 a practicante-profesor en la Universidad de Caldas, 12/02/2018)

[...] se eles fossem um pouco mais nas escolas, vissem mais como é que está a situação atual nas escolas eu acho que eles mudariam, porque a gente chega meio que moldados pelos professores. Chegamos querendo pôr em prática tudo o que a gente viu, e aí acaba não dando certo, acaba se frustrando [...] aí às vezes as contribuições que o professor dava eu achava extremamente inviável. Então eu acho que eles querem impor uma teoria que, às vezes, eu particularmente não concordava [...] porque eu acho que falar é uma coisa e ir lá e atuar é outra. Então eles ficam muito no texto, na literatura e acaba esquecendo um pouco de ir à prática também. (Entrevista 5 a practicante-profesor en la Universidad Estadual Paulista, 20/08/2014)

El asesor que tengo hoy día, la verdad no me da mucho asesoramiento, sino que él lo hace más desde la parte 
disciplinar, en la que tiene que ser todo controlado; [...] el asesor lo ve a uno como una amenaza porque está es pendiente de lo disciplinar, no podemos hacer nada como medio divertido porque entonces él ahí mismo nos va a juzgar. (Entrevista 2 a practicante-profesor en la Universidad de Caldas, 17/02/2018)

En ese sentido, se entiende que el profesor-asesor durante el prácticum se centra en su gran mayoría en aspectos disciplinares; por ejemplo, el acompañamiento es esporádico, la retroalimentación insuficiente, poca reflexión, y generalmente se basan en lo establecido por las directrices reglamentarias, alejándose de la realidad del contexto y las necesidades de los practicantes-profesores.

Por ello, es necesario que el profesor-asesor incluya en su quehacer docente y profesional que el practicante-profesor realice reflexiones sobre su práctica docente, fortalezca los saberes docentes, facilite el desarrollo emocional, etc., y así potencie la formación integral en el trascurso del prácticum.

Como encerramiento provisional, apostamos a que el profesorasesor en los programas de Licenciatura en Educación Física, Recreación y Deporte en Colombia y Brasil pueda, a través de las experiencias teórico/prácticas, trasformar los contextos educativos, pedagógicos y didácticos en el prácticum, mediante la trasformación de las realidades al formar las interacciones constantemente con el practicante-profesor y el profesor-titular con el uso del feedback (retroalimentación) intrínseco y extrínseco; además, con la empatía, asertividad, creatividad, reflexión y utilización del cuerpo como colectividad biológico/anatómica y simbólica que piensa y siente, con el fin de trasformar los conocimientos, los sentimientos y conocer los fines del prácticum. 


\section{Conclusiones}

En el marco de nuestra intencionalidad como investigadores en la formación de los practicantes-profesores en los programas de la Licenciatura en Educación Física que contemplan la PPD y el ECS, respectivamente en la Universidad de Caldas en Colombia y en la Universidad Estadual Paulista en Brasil, consiste en comprender desde la mirada de los practicantes-profesores al profesor-asesor en el proceso de formación en el prácticum, se hace importante presentar reflexiones sobre las características, legislaciones, directrices, así como sobre las necesidades de cada contexto educativo en el cual se desarrolla el prácticum.

En el prácticum es fundamental la labor del profesor-asesor, ya que es el responsable de acompañar, guiar, supervisar, asesorar, planificar, organizar, orientar, gestionar, controlar y evaluar al y con el practicante-profesor en el contexto educativo del prácticum, con el objetivo de que este, en su quehacer profesional, se haga consciente de, y que los identifique y fortalezca, los saberes docentes adquiridos desde su propio estilo de enseñanza/aprendizaje.

Es claro que la función más importante para el profesor-asesor es buscar que el practicante-profesor logre los objetivos previamente establecidos; sin embargo, en la mayoría de las ocasiones estos se concentran más en alcanzar los estándares cuantitativos y los objetivos disciplinares asignados por los programas de formación, lo cual podrá afectar el rendimiento de los practicantesprofesores en su formación integral.

El profesor-asesor, durante el prácticum, también tendrá el reto de desarrollar las capacidades, habilidades y expresiones del practicante-profesor, mediante retroalimentaciones que fomenten la crítica objetiva y la corrección constante de posibles errores presentados. Por tal razón, en el proceso del prácticum el profesorasesor deberá formar con mirada global, una en que se incluya a la comunidad (estudiantes, padres de familia, administrativos, vecinos en general, etc.) en las dinámicas de enseñanza y aprendi- 
zaje y en el desarrollo de habilidades, competencias, experticias y maestrías (Acosta y Vasco, 2013) para el desarrollo de la sociedad.

Por ello, en la universidad y la escuela se necesita un nuevo modelo de actores educativos en el campo de la educación física, recreación y deporte, para que no contemple solamente la formación académica, sino también la capacidad de situar la reflexión de los contextos educativos, formar la personalidad, las emociones y las prácticas corporales que envuelven la realidad educativa a partir de sus historias de vida. Esto se logra a través de interacciones comunicativas entre los actores que se encargan de enfrentar los desafíos teórico/prácticos con el fin de fortalecer el acompañamiento, las condiciones profesionales, las potencialidades y el perfeccionamiento del proceso de enseñanza/aprendizaje en la comunidad educativa.

Como conclusión parcial, se propone seguir realizando investigaciones que permitan comprender profundamente el proceso del prácticum en la clase de educación física, desde la generación de estrategias que contribuyan al desarrollo de las mejores prácticas docentes y permitiendo que el maestro en formación (practicanteprofesor) utilice los mejores enfoques y escenarios para facilitar que las políticas educativas y las realidades escolares contribuyan al mejoramiento de la calidad educativa.

\section{Agradecimientos}

Se agradece a la Universidad de Caldas y a la Universidad Estadual Paulista, en sus programas de Licenciatura en Educación Física, Recreación y Deporte, así como a los practicantes-profesores por disponer de su tiempo y dialogar sobre su prácticum (Práctica Docente), y por las perspectivas sobre el profesor-asesor, lo cual hizo posible la ruta y la culminación de este ejercicio investigativo en Colombia y Brasil. 


\section{Referencias bibliográficas}

Acosta, D. A. y Vasco, C. E. (2013). Habilidades, competencias y experticias: más allá del saber qué y el saber cómo. Bogotá: Corporación Universitaria Unitec, Universidad de Manizales y Fundación Centro Internacional de Educación y Desarrollo Humano (Cinde).

André, M. E. D. A de. (2013). O que é um estudo de caso qualitativo em educação? Revista da FAEEBA - Educação e Contemporaneidade, 22(40), 95-103.

Ausubel, D. P. (2002). Adquisición y retención del conocimiento: una perspectiva cognitiva. Barcelona: Paidós.

Ayala, C. F. (2015). Pedagogical Guidelines on Supervised Internship of Physical Education in Colombia, Brasil, Argentina and Chile (tesis doctoral). Institute of Biosciences, University State St Paulo, Rio Claro, Brasil.

Ayala, C. F., Souza, S. de y laochite, R. T. (2015). Formación de profesores: encuentros entre Colombia, Brasil y Chile. Revista de Investigaciones, 15(26), 18-33. Recuperado de http://www.revistas.ucm.edu.co/ojs/ index.php/revista/article/view/41

Ayala, C. F., Souza, S. de y laochite, R. T. (2017). Políticas docentes de formação de professores no estágio supervisionado: Colômbia, Brasil, Argentina e Chile. São Paulo: Cultura Acadêmica Editora.

Ávila, R. (2019). La fabulosa historia del atletismo colombiano. Bogotá: QuadGraphics.

Betancur, J. E., López, C. R. y Arcila, W. O. (2018). El docente de educación física y sus prácticas pedagógicas. Revista Latinoamericana de Estudios Educativos, 14(1), 15-32.

Bisquerra, R. (2016). 10 ideas clave: educación emocional. Barcelona: Graó. 
Bolívar, R. M. (2019). Investigar la práctica pedagógica en la formación inicial de maestros. Pedagogía y Saberes, 51, 9-22.

Calle, M. G., Remolina, N. y Velásquez, B. M. (2011). Incidencia de la inteligencia emocional en el proceso de aprendizaje. NOVA - Publicación Científica en Ciencias Biomédicas, 15(9), 94-106.

Cárdenas, O. L. y Cárdenas, M. C. (2019). La historia de los saberes artísticos en la escuela pública colombiana en la primera mitad del siglo XIX. Pedagogía y Saberes, 50, 173-185.

Congreso de Colombia. (8 de febrero de 1994). Ley General de Educación (Ley 115 de 1994). Recuperado de https://www.mineducacion.gov. co/1621/articles-85906_archivo_pdf.pdf

Conselho Nacional de Educação. (2002). Resolução CNE/CP 1, de 18 de fevereiro de 2002. Institui Diretrizes Curriculares Nacionais para a Formação de Professores da Educação Básica, em nível superior, curso de licenciatura, de graduação plena. Recuperado de http://portal.mec. gov.br/cne/arquivos/pdf/rcp01_02.pdf

Corrales, N., Ferrari, S., Gómez, J. y Renzi, G. (2010). La formación docente en educación física. Buenos Aires: Noveduc.

Correa, E. (2011). La práctica docente: una oportunidad de desarrollo profesional. Perspectiva Educacional, 50(2), 77-95. Recuperado de http://www.perspectivaeducacional.cl/index.php/peducacional/article/ viewFile/41/21

Díaz, L., Torruco, U., Martínez, M. y Varela, M. (2013). La entrevista, recurso flexible y dinámico. Investigación en Educación Médica, 2(7), 162-167. Recuperado de http://www.redalyc.org/articulo.oa?id=349733228009

Fuzii, F. T. (2010). Formação de professores de educação física e avaliação: investigando a restruturação curricular dos cursos de licenciatura (tesis de maestría). Instituto de Biociências, Universidade Estadual Paulista, Rio Claro, Brasil. 
Freire, P. (1994). Cartas a quien pretende enseñar. Ciudad de México: Siglo XXI.

Gómez, O. Y. A., \& Ostos, O.-L. (2018). El constructivismo y el construccionismo. Revista Interamericana de Investigación, Educación y Pedagogía, RIIEP, 11(2), 115-120.

Herrera, L., Buitrago, R. y Ávila, A. (2016). Empathy in Future Teachers of the Pedagogical and Technological University of Colombia. Journal of New Approaches in Educational Research, 5(1), 30-37. DOI: https://doi. org/10.7821/naer.2016.1.136

Lobo, L. y Quintero, B. (2016). El rol del supervisor escolar en centros educativos de un municipio del estado Mérida - Venezuela. Administración Educacional, 4(4), 103-127.

Martínez, P. S. (2019). El prácticum en el grado de Pedagogía: en busca de una comunidad de prácticas. Revista Interamericana de Investigación, Educación y Pedagogía, 12(1), 43-54. DOI: https://doi.org/10.15332/ s1657-107X.2019.0001.07

Martínez, E. y Raposo, M. (2010). Funciones generales de la tutoría en el prácticum: entre la realidad y el deseo en el desempeño de la acción tutorial. Revista de Educación, 354, 155-181.

MEN (Ministerio de Educación Nacional). (2010). Documento n. 15. Orientaciones pedagógicas para la educación física, recreación $y$ deporte. Recuperado de https://www.mineducacion.gov.co/1759/ articles-340033_archivo_pdf_Orientaciones_EduFisica_Rec_Deporte. pdf

MEN (Ministerio de Educación Nacional). (2017). Resolución 18583 de 2017. Recuperado de http://legal.legis.com.co/document/Index?obra=I egcol\&document=legcol_00821d7db80a4391b82274489461d53b

Ocampo, Á. (2009). De la doctrina a la escuela: una historia de la educación en Manizales y Caldas. Manizales: Manigraf. 
Paulilo, M. A. (1999). Pesquisa qualitativa e a história de vida. Revista Serviço Social, 2(2), 135-148.

Portela, G. H., Taborda, Ch. J. y Loaiza, Z. Y. E. (2017). El currículum en estudiantes y profesores de los programas de formación de educadores de la Universidad de Caldas de la ciudad de Manizales: significados y sentidos. Revista Latinoamericana de Estudios Educativos (Colombia), 13(1), 17-46.

Presidência da República. (1977). Lei n.ㅇ 6494, de 7 de dezembro de 1977. Dispõe sobre o estágio de estudante de estabelecimentos de ensino superior e de 2ㅇ Grau Regular e Supletivo, nos limites que especifica e dá outras providências. Recuperado de http://www.planalto.gov.br/ ccivil_03/leis/L6494.htm

Presidência da República. (1998). Lei n. 9696, de 1 setembro de 1998. Dispõe sobre a regulamentação da profissão de Educação Física e cria os respectivos Conselho Federal e Conselhos Regionais de Educação Física. DOU, 2 set 1998.

Presidência da República. (2008). Lei n. 11788, de 25 de setembro de 2008. Dispõe sobre o estágio de estudantes. DOU, 26 set 2008. Recuperado de http://www.planalto.gov.br/ccivil_03/_ato2007-\%20 2010/2008/lei/l11788.htm

Saiz, A. y Susinos, T. (2016). "Nos dabas la confianza para hablar". El supervisor universitario en un prácticum reflexivo. Revista Iberoamericana sobre Calidad, Eficacia y Cambio en Educación, 15(1), 55-69. DOI: http://dx.doi.org/10.15366/reice2017.15.1.004

Santos, M. (2006). Arqueología de los sentimientos en la escuela. Buenos Aires: Bonum.

Schön, D. (2002). La formación de profesionales reflexivos: hacia un nuevo diseño de la enseñanza y el aprendizaje en las profesiones. Barcelona: Paidós. 
Simões, J. L. y Silva, I. T da. (2013). A educação física no Brasil. Revista Electrónica Actividad Física y Ciencias, 5(1).

Soler, E. (2001). La supervisión educativa en sus fuentes. Madrid: Santillana.

Souza Neto, S. de. (2014). A formação de professores de Educação Física no Brasil: Novos rumos, novos desafios. En A. Albuquerque, C. Pinheiro y R. Resende (eds.), A formação de formadores em Educação Física e Desporto: perspectivas internacionais, tendências atuais (pp. 237-254). Maia: Edições ISMAI.

Strauss, A. y Corbin, J. (2002). Bases de la investigación cualitativa: técnicas y procedimientos para desarrollar la teoría fundamentada. Medellín: Universidad de Antioquia.

Taborda, Ch. J. (2017). Aspectos pedagógicos y didácticos asociados al desarrollo de la flexibilidad en niños y niñas. Manizales: Editorial Universidad de Caldas.

Tardif, M. (2010). Saberes docentes e formação profissional. São Paulo: Vozes.

Torrijos, P., Martín, J. y Rodríguez, M. (2018). La educación emocional en la formación permanente del profesorado no universitario. Profesorado. Revista de Currículum y Formación del Profesorado, 22(1), 579-597.

Wood, P. y Smith, J. (2018). Investigar en educación: conceptos básicos y metodología para desarrollar proyectos de investigación. Madrid: Narcea.

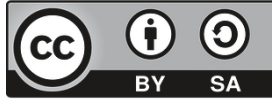

